\title{
Techniques in High-Resolution Coincidence Counting
}

\author{
George H. Minton ${ }^{1}$
}

\begin{abstract}
Circuitry, svstems, and techniques used in radiation coincidence measurements with millimicrosecond resolution are discussed. An analysis of the time fluctuations in a scintillation counting system is presented and comparisons with experimental results are made.
\end{abstract}

\section{Introduction}

The applications of coincidence systems to problems in nuclear research are of two types. In one, the coincidence system is used in order to detect events that are coincident in time. In the other, it is applied to the measurement of the distribution in time of noncoincident events. The characteristics of some coincidence systems have been studied in order to determine their suitability for such applications. The factors that have been considered are (1) the resolution obtainable with available radiation detectors, (2) the relationship between the resolution and the efficiency of the system., (3) the factors that determine the response of the system. to coincident events and to events separated in time by intervals of the order of magnitude of the resolving time or less.

Although the ideal coincidence system will respond only to events that are exactly coincident, such response is not obtainable in practice. The events involved are signaled by the production in the detector of electrical pulses - often widely varying in amplitude - and the resolution of the system is determined by the response of the detector and that of the circuit to which the signals are fed. Spurious response of such a system corresponds to the recording as coincidences of single pulses or of events that are more closely separated in time than its resolution. As the number of the latter events in any given time interval depends on the product of the counting rates in the individual detectors, the resolving time of the system often provides a limitation on the permissible counting rates. Failure of the system to respond to coincident events can be measured in terms of an efficiency factor that is less than or equal to 1 .

In the measurement of the distribution in time of noncoincident events, the signal from one detector is delayed before being fed into the coincidence circuit, and the number of coincidences recorded is studied as a function of the length of the inserted delay time. These data furnish a response curve of the system for the events under study. If the rate of occurrence of events does not vary appreciably in a time of the order of the resolution of the system. the shape of the response curve of the system to coincident events is not critical. On the other hand, for studying more rapidly varying distributions it

${ }_{1}^{1}$ Present address, Westinghouse Electric Corporation, Pittsburgh, Pa. is necessary to obtain a response curve of the system for coincident events. The resolution of the system is often defined as one-half the time as measured on the delay axis between points corresponding to one-half the maximum counting rate of such a response curve. By proper analysis of this curve along with that for noncoincident events, it is possible to measure time intervals to an order of magnitude smaller than the resolution of the system, as defined above, to an accuracy of the order of 10 percent.

\section{Detectors}

The only type of radiation detector presently available that is suitable for very fast coincidence work is the scintillation counter. Among the various phosphors available, there is a wide divergence among the values of the time constants for the emission of light. In general, the organic phosphors emit their light in one-tenth to one one-hundred th the time of the inorganic materials, so that attention will be confined to the scintillation counter with organic phosphor. The measurements reported here were made, using RCA 5819 and 6199 photomultipliers with stilbene crystals or solutions of terphenyl in toluene as scintillators.

\section{Coincidence Circuit}

The coincidence circuit receives the pulses from. the detectors and provides an output pulse whenever the input pulses are in coincidence. The ideal coincidence circuit never responds to single pulses, regardless of their amplitude, and never registers a coincidence when the input pulses are separated by a time greater than their own duration. The circuit should have as low an input capacitance as possible in order to minimize any effect on the rise time of the input pulse. The requirement of low capacitance is most satisfactorily met by the use of a circuit constructed from crystal diodes. Figure 1 is a schematic diagram of such a circuit-a modification of that of DeBenedetti and Richings [1] ${ }^{2}$ - which has been used in this investigation. Study of the response of this circuit to pulses from a mercury-relay pulse generator and from scintillation counters has revealed that the response of the circuit is not a limiting factor in the operation of the coincidence systems using nuclear radiation detectors, so that an extensive comparison

${ }^{2}$ Figures in brackets indicate the literature references at the end of this paper. 


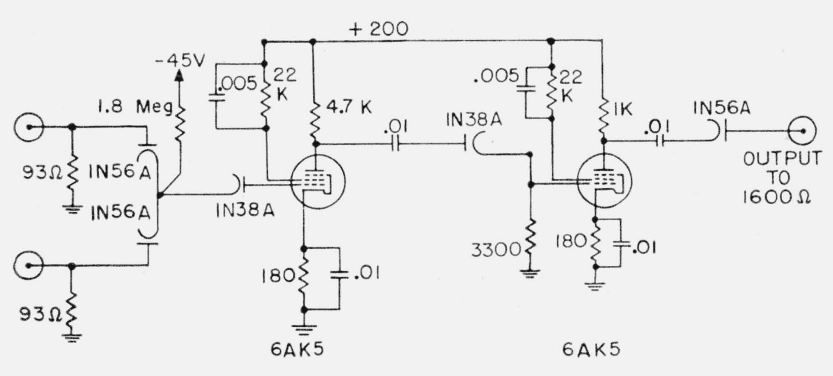

FigURE 1. High-resolution coincidence circuit for negative pulses from the anodes of two photomultiplier tubes.

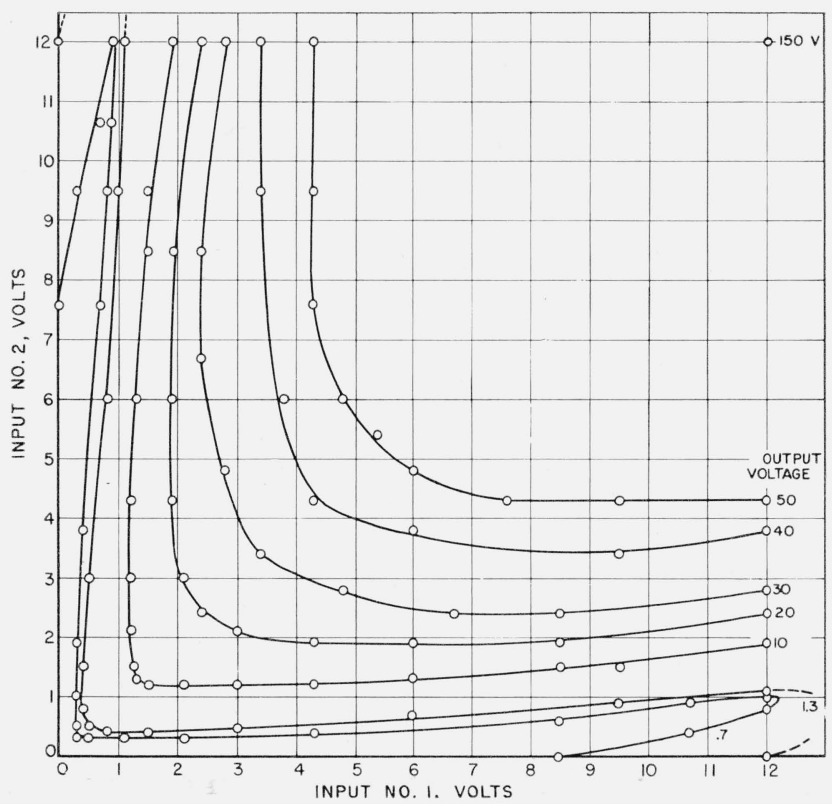

Figure 2. Response of the fast coincidence circuit of figure 1 and associated amplifier to simultaneous pulses from a mercuryrelay pulse generator.

Fast-rising negative pulses having an exponential decay time of $2.5 \times 10^{-9} \mathrm{sec}$ were generated by discharging a capacitor through the relay into a coaxial cable. The pulses were further shaped, as in the scintillation counters, by reflection in 5 -in shorted stubs of RG 62-U coaxial cable.

of available coincidence circuits has not been made. On the basis of published information, however, it is believed that this circuit is at least as good as any other in use today. Figures 2 and 3 illustrate the response of this circuit to pulses from the mercuryrelay pulse generator. In order to closely approximate the shape of the pulse from a scintillation counter with liquid scintillator, pulses having a rise time characteristic of the mercury switch and an exponential decay of $2.5 \mathrm{~m} \mu \mathrm{sec}$ were used. It is interesting to note that when the two input pulses are of different amplitudes the response of the circuit is nearly independent of the amplitude of the larger pulse. From the curve of figure 2 corresponding to $1.3-\mathrm{v}$ output, it can be seen that the ratio of the amplitudes of a pair of equal coincident pulses to the amplitude of a single pulse producing the same response is about $1: 25$. The width of the curves of voltage versus signal delay is determined by the duration of the input. Higher pulses appear wider

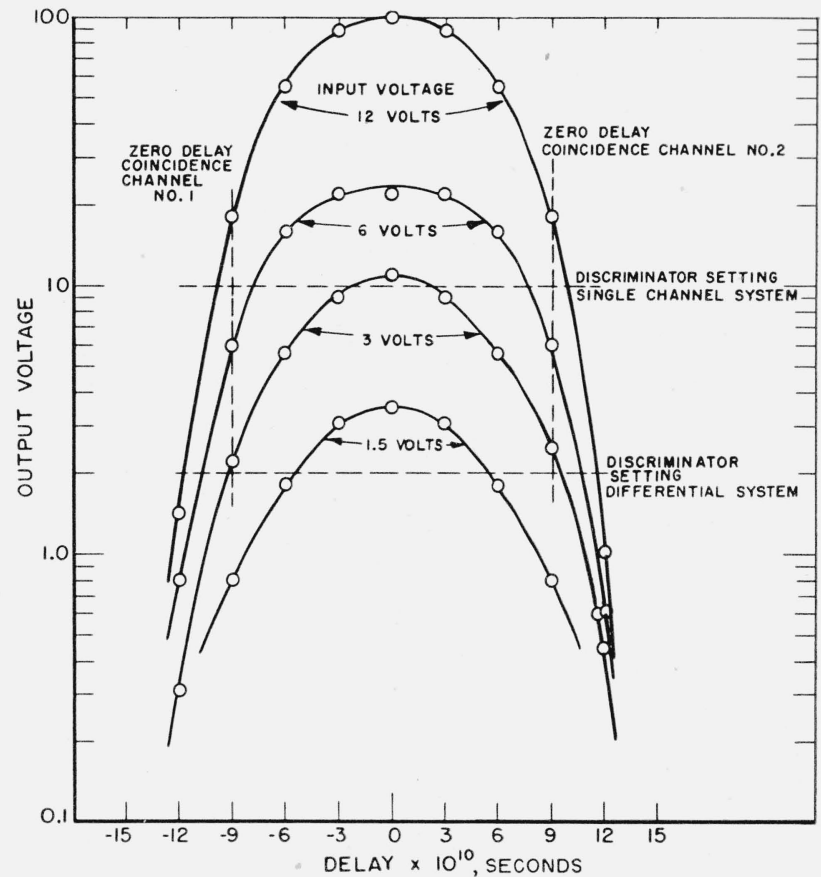

FIgure 3. Response of the coincidence circuit of figure 1 and associated amplifier as a function of the time separation of a pair of equal amplitude pulses from the mercury-relay pulse generator.

The dashed lines refer to operation of the circuit as part of a differential coincidence system (see text).

because of their finite rate of rise and exponential decay.

In the experiments to be described, the photomultiplier voltages were adjusted so that pulse amplitudes from 1 to $5 \mathrm{v}$ were produced at the coincidence circuit as a result of absorption in the scintillator of Compton electrons having energies between 200 and $1,000 \mathrm{kev}$.

\section{Coincidence System}

In the detection of gamma rays with organic scintillators, the energetic electrons produced in the Compton scattering of the gamma ray excite the phosphor, and the process of deexcitation results in the emission of light, which is detected by the photomultiplier. The amount of light yield due to the interaction of a particular gamma ray depends on the energy of the scattered electron. Thus, by the nature of the Compton process, a monoenergetic gamma ray produces a continuum of pulse amplitudes at the output of the photomultiplier, with maximum pulse height depending on the gamma energy.

As the response of the coincidence circuit depends on the amplitude of the input pulse, it is desirable to work with pulses above some minimum. amplitude. This can be accomplished without affecting the speed of response of the coincidence circuit by gating its output with the pulses from an amplitude discriminator into which the amplified pulses from the last dynode of the photomultiplier are fed. Because the pulse distribution from the Compton electrons will 
have a well-defined maximum determined by the energy of the original gamma ray, one can study the response of the system to as narrow a band of pulse amplitudes as desired in the neighborhood of the maximum with the use of an integral pulse-height selector. If one desires to work with pulse amplitudes below the maximum of the Compton distribution, a differential pulse-height analyzer is required. For this study, integral pulse-height selectors were used with two sources, $\mathrm{Na}^{22}$ with annihilation radiation coincidences of $0.510-\mathrm{Mev}$ gamma rays and $\mathrm{Co}^{60}$ with coincidences between a 1.17 - and a $1.33-\mathrm{Mev}$ gamma ray.

The resolution of the coincidence system as a whole is dependent on two factors. The first is the time distribution of pulses produced by the two photomultiplier tubes when the original gamma rays are truly coincident. The second factor is the duration of pulses from the photomultiplier tube because the coincidence circuit will respond to pulses that are delayed relative to one another if the delay is less than the duration of the pulses.

The usual procedure for studying the response of a coincidence system is to set up two scintillation counters, to excite the phosphors with coincident gamma rays such as those resulting from annihilation of positrons, and to study the number of coincidences recorded as a function of an artificial delay inserted in the system by varying the length of cable from one of the detectors to the coincidence circuit. If the pulses were square, of fixed duration, and always coincident in time, the coincidence rate would be constant as the cable length was varied over a range corresponding to twice the duration of the pulse, and would be zero outside this range. On the other hand, if the pulse width were negligible, the curve of coincidence rate versus inserted delay would correspond exactly to the time distribution of the pulses from the detectors.

The first step toward obtaining good resolution was the choice of the organic scintillating materials. One of the fastest known crystalline phosphors is trans-stilbene, which responds to a Compton electron by emission of an exponential pulse of light with a decay constant of about $6 \times 10^{-9} \mathrm{sec}$. This was the phosphor that was chosen for the initial tests. Next, a series of tests was run on a number of RCA 5819 photomultiplier tubes in order to select a few that were capable of producing pulses large enough to actuate the coincidence circuit without amplification. Some of the tubes tested were capable of operation in the neighborhood of $2,000 \mathrm{v}$, and exhibited a maximum gain about 10 times that of the average tube. Two of these better tubes were chosen for use in the coincidence system. In this way, rise-time limitations introduced by amplifiers were eliminated. The duration of the pulse at the output of the photomultiplier tube can be reduced by using a shorted stub of coaxial cable to produce a delayed canceling pulse of opposite sign. The minimum pulse duration obtainable by this procedure is approximately equal to the rise time of the original pulse. This rise time depends primarily on the photomultiplier tbue, and appears to be 2 to $3 \times 10^{-9}$ sec if the time constant of the output circuit is less than this value.

As the best resolutions commonly attained with scintillation counters are of this order of magnitude, it appears that the duration of the input pulses and fluctuations therein may often be limiting factors that mask the effects of the time fluctuations in the pulses produced by coincident radiation events. A glance at figure 3 suggests a method to further reduce the effect of pulse duration upon the resolution of the system. Because of the width of the pulses, the rate of change of output voltage with delay is small in the region around zero delay. For this reason it is desirable to operate the circuit with such cable lengths that simultaneous pulses are delayed relative to one another by about $1 \mathrm{~m} \mu \mathrm{sec}$ so that small fluctuations in the time of occurrence of pulses will produce a measurable change in the output voltage. In order to have a symmetric system, it is necessary to use two fast coincidence circuits operating on opposite sides of the maximum of the coincidence resolution curve, as indicated by the vertical dashed lines in figure 3 . The outputs of these two circuits are fed to a slow coincidence circuit. This complete unit is then referred to as a differential coincidence circuit, in analogy with a system proposed by Bay [2]. The two individual fast coincidence circuits will be referred to as channels of the differential coincidence circuit.

A coincidence is recorded only when there are simultaneous output pulses from the two fast coincidence circuits. The cabling from the detectors is so chosen that in the event of a true coincidence the pulse from one detector arrives before the pulse from the other detector at channel 1 and after the pulse from the other detector at channel 2 .

As an illustration, it is useful to consider the response of the circuit to square pulses of fixed amplitude. By making the differences in transmission time just less than the duration of the pulse, all incidents of simultaneity of initial pulses result in a coincidence being recorded, whereas even a delay that is less than the duration of the pulses results in a rejection by the differential circuit. The situation is shown schematically in figure 4 . If the pulse from detector 2 is early, there will be no coincidence recorded in channel 1 , and if it is late, there will be no coincidence recorded in channel 2. Both channels will produce an output pulse only in case of a true coincidence.

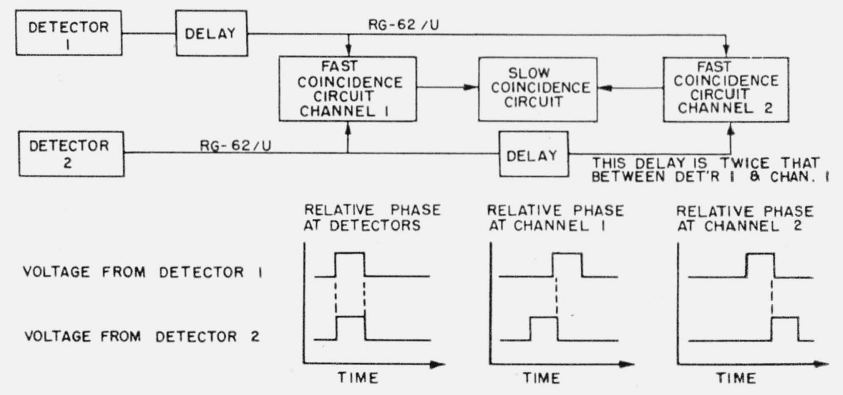

Figure 4. Principle of the differential coincidence technique as used in this investigation. 
A block diagram of the differential coincidence circuit is shown in figure 5 . The response of the system of coincident radiation is illustrated in figures 6,7 , and 8 . The curves of figure 6 show the response of the individual channels to coincidences from cobalt-60. The displacement of the curves is to be attributed to the cabling arrangement between channels. The coincidence circuits are 100-percent efficient for pulses above the levels of the gating pulse-height selectors. The width of the curves is principally due to the width of the pulses from the photomultipliers.

Figure 7 shows the response when the two circuits are operated as a differential unit with the inserted delays such that there is no loss in efficiency. The fact that there is no loss in efficiency indicates that for zero delay the pulses overlap sufticiently at both channels to compensate for any time fluctuations that are introduced by the detectors. If the differential cabling between channels is increased, there will be a decrease in the maximum coincidence rate when the time during which both pulses are present at the inputs of the coincidence circuit becomes less than the fluctuations in time of occurrence of the pulses. Figure 8 is a response curve for the system when this differential delay is sufficient to reduce the maximum coincidence rate to 10 percent of its previous value. The shape of this curve is determined primarily by the time fluctuations of pulses from the detectors, and its width is a measure of the magnitude of these fluctuations. This conclusion is confirmed by the fact that a reduction in efficiency below about 20 percent results in no appreciable further narrowing of the response curve, indicating that the "channel width" in time is then much narrower than the distribution in time of the pulses arriving at the coincidence unit. A measure of the channel width is the quotient of the random coincidence rate by the product of the individual counting rates of the detectors, which, under the conditions of figure 8 , is $2.8 \times 10^{-10}$ sec.

Another factor that contributes to the width of the response curve is the variation in amplitude of the pulses at the input of the coincidence circuit. This effect has been minimized in this study by limiting the amplitude variation to about 25 percent with the gating pulse-height selectors.

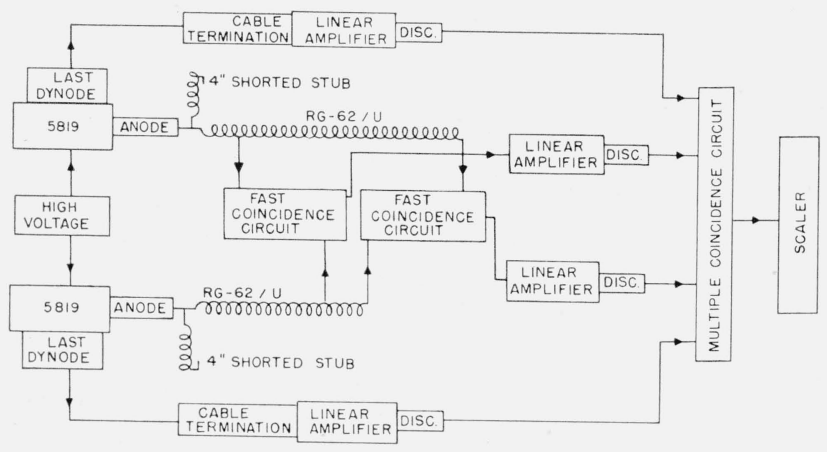

FIGURE 5. Block diagram of the differential coincidence system with gating pulse height selectors.

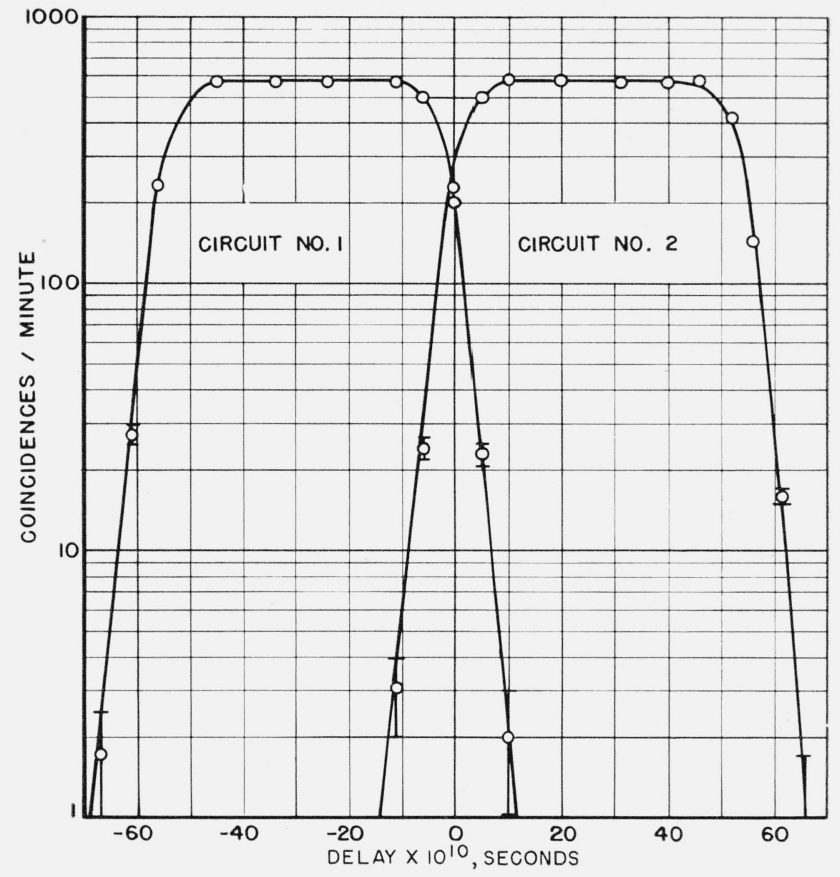

Figure 6. Response of the individual channels of the coincidence system of figure 5 to pulses produced by coincident $\mathrm{Co}^{60}$ gamma rays on stilbene with 5819 photomultipliers.

Different fixed lengths of cable interconnecting the inputs of the two circuits account for the displacement of the centroids of the two curves. The delay is introduced by varying the cable lengths from the photomultipliers.

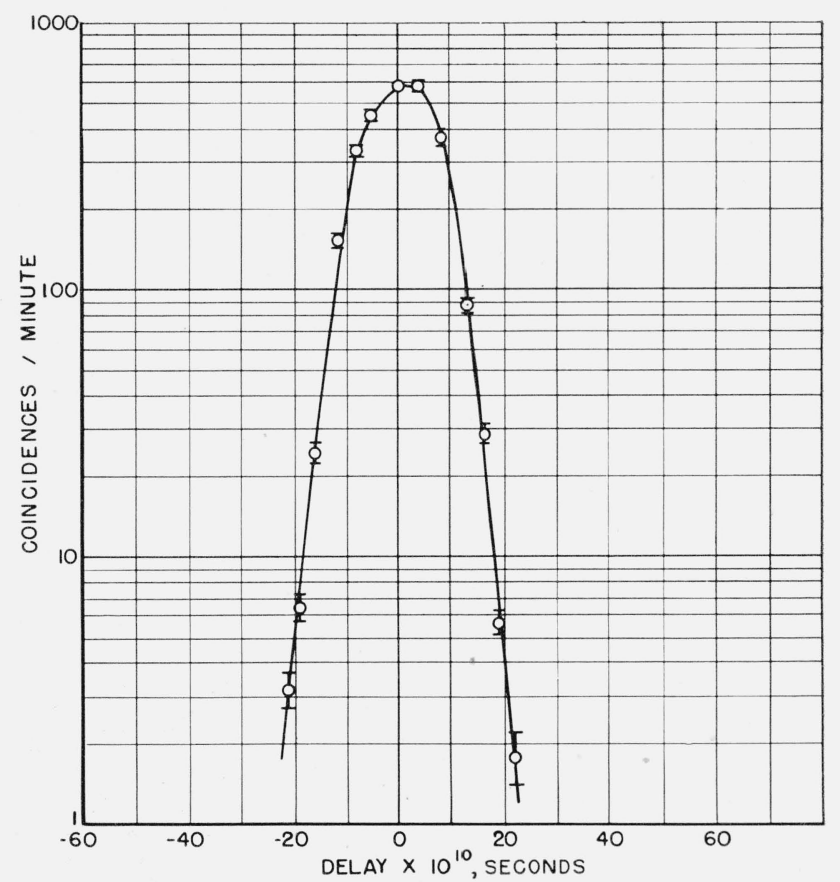

Figure 7. Response of the differential coincidence system of figure 5, with cabling so arranged that the system responded to all coincident gamma rays producing pulses with amplitudes above the levels of the gating pulse-height selectors.

5819 photomultipliers and stilbene crystals were used with $\mathrm{Co}^{60} \gamma$-radiation. 


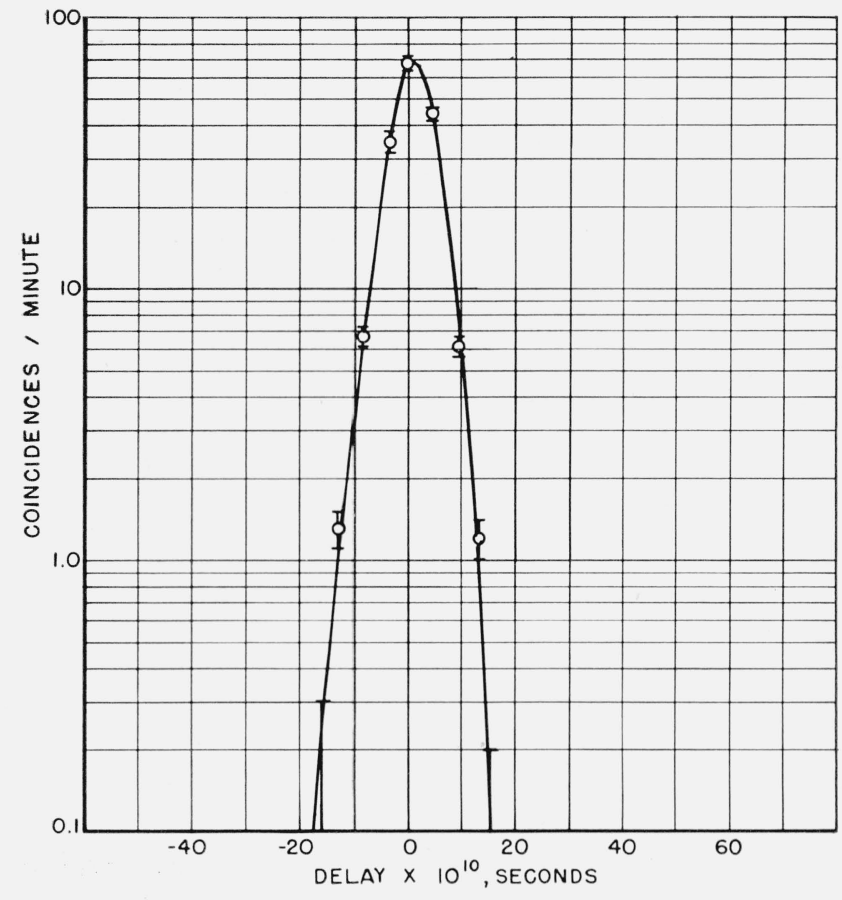

Figure 8. Response of the differential coincidence system of figure 5, with cabling arranged so that the system responded to 10 percent of the coincidences between gamma rays producing pulses above the level of the gating pulse-height selectors.

The width of this curve is a measure of the time fluctuations in the scintillation counter.

5819 photomultipliers and stilbene crystals were used with $\mathrm{C}^{60} \gamma$-radiation.

\section{Time Fluctuations in the Scintillation Counter}

\subsection{Theoretical Analysis}

The differential coincidence circuit has been used to study the distribution in time of pulses from the photomultipliers under various operating conditions. Two sources of coincident radiation were utilized. $\mathrm{Na}^{22}$, a positron emitter, was used to obtain the coincident $0.510-\mathrm{Mev}$ gamma rays of annihilation. The number of unwanted coincidences between the $1.3-\mathrm{Mev}$ gamma rays also emitted by this source and the gamma rays of annihilation was made negligible by reducing the solid angles intercepted at the source by the detectors. Because the gamma rays of 2quantum annihilation are emitted in exactly opposite directions, their coincidence rate depends only on a smaller of the two solid angles, whereas the rate of the coincidences involving the $1.3 \mathrm{-Mev}$ gamma rays diminishes as the product of the solid angles of the two detectors. A source of coincidences between higher-energy gamma rays is $\mathrm{Co}^{60}$, which emits a beta particle followed by 1.17 - and $1.33-\mathrm{Mev}$ gamma rays in coincidence.

Because the apparent time distribution of pulses from the detectors is determined by fluctuations in the rise times of the pulses, the factors that influence the rise time will be the factors that influence the o bserved distribution. These factors are fluctuations in photon emission and collection time and fluctuations in transit time of electrons in the photomultiplier. Post and Schiff [3] have discussed the statistical limitations on the resolving time of a scintillation counter imposed by the fluctuations in photon arrival time, and Morton [4] has reported the results of a study in which account also has been taken of the transit-time fluctuations in the photomultiplier. Following the treatment of Post and Schiff, we consider a phosphor which, upon excitation at $t=0$, emits photons in an exponential decay process, so that

$$
f(t)=\int_{0}^{t} R \lambda e^{-\lambda t} d t
$$

where $f(t)$ is the average number of photoelectrons produced at the photocathode in the time interval $0-t, R$ is the total number of photoelectrons produced as a result of the excitation of the phosphor, and $\lambda$ is the decay constant of the phosphor.

$$
f(t)=R\left(1-e^{-\lambda t}\right) \simeq R \lambda t,
$$

where $t \ll 1 / \lambda$.

The number, $f(t)$, will be subject to statistical fluctuations described by the Poisson distribution, so that the probability of $N$ electrons being produced in the interval $0-t$ is

$$
P_{N}(t)=\frac{[f(t)]^{N} e^{-f(t)}}{N !} .
$$

The probability that the Qth photoelectron is produced between $t$ and $t+d t$ is

$$
W_{Q}(t)=P_{Q-1}(t)[d f / d t] d t .
$$

The variance $(v)$ in time of production of the Qth photoelectron is $\overline{t^{2}}-(\bar{t})^{2}$.

$$
\begin{aligned}
v & =\int_{0}^{\infty} t^{2} W_{Q}(t) d t-\left[\int_{0}^{\infty} t W_{Q}(t) d t\right]^{2} \\
& \simeq R \lambda \int_{0}^{\infty} \frac{t^{2}[R \lambda t]^{Q-1} e^{-R \lambda t}}{(Q-1) !} d t-\left[R \lambda \int_{0}^{\infty} \frac{t[R \lambda t]^{Q-1} e^{-R \lambda t}}{(Q-1) !} d t\right]^{2} \\
& \simeq Q / R \lambda)^{2} .
\end{aligned}
$$

Post and Schiff have considered the problem more generally, and obtain, for $\lambda t<1$,

$$
v(Q)=\frac{Q}{(R \lambda)^{2}}\left[1+\frac{2(Q+1)}{R}+\text { higher-order terms in } \frac{Q}{R}\right] \text {. }
$$

To develop a model with which to compare the experimental results, $Q$ was designated as the average number of photoelectrons that would be produced in a time equal to the average rise time of the output pulse. It is assumed that the variance in the time of response of the system to the pulse is given by the arithmetic mean of the variances associated individually with the first $Q$ electrons. This assumption 
is reasonable, because the pulses are essentially differentiated by the use of the shorted stub, and the differential coincidence technique involves a coincidence between the leading edge of one derivative pulse and the trailing edge of the other. The average variance will be called $\vec{v}_{1}(Q)$.

$$
\begin{aligned}
\bar{v}_{1}(Q) & \left.=\frac{1}{(R \lambda)^{2}}\left[\frac{1}{Q} \sum_{N=1}^{Q}\left\{N+\frac{2}{R} N+N^{2}\right)\right\}\right] \\
& =\frac{1}{(R \lambda)^{2}}\left[\frac{Q(Q+1)}{2 Q}+\frac{2(Q+1)(Q+2)}{3 R}\right] \\
& \simeq \frac{Q}{2(R \lambda)^{2}}+\frac{2 Q^{2}}{3 R(R \lambda)^{2}},
\end{aligned}
$$

where $Q \gg 1$.

The time of output of the signal will also depend on the fluctuations in electron transit time in the photomultiplier. Assuming, as did Morton, that in each stage the transit times can be described by a Gaussian distribution:

$$
P\left(t_{i}\right)=\frac{1}{\beta_{i} \sqrt{2 \pi}} \exp \left\{-\frac{\left(t_{i}-t_{o i}\right)^{2}}{2 \beta_{i}^{2}}\right\},
$$

where $t_{o i}$ is the average transit time in the $i$ th stage, and $\beta_{i}$ is a constant determined by the geometry and voltage in the $i$ th stage. Let $n_{i}$ be the average number of electrons in the $i$ th stage due to $Q$ electrons at the photocathode. The variance in the average transit time of these electrons will be $\beta_{i}^{2} / n_{i}$. If $\sigma$ is the average gain per stage, then $n_{i}=Q \sigma^{(i-1)}$. The average total transit time of the secondary electrons due to one photoelectron is $\Sigma t_{o i}$, and the variance in this quantity, when $Q$ photoelectrons are involved, is

$$
\bar{v}_{2}=\sum_{i} \frac{\beta_{i}^{2}}{n_{i}}=\sum_{i} \frac{\beta_{i}^{2}}{Q \sigma^{(i-1)}}=\frac{1}{Q} \sum_{i} \frac{\beta_{i}^{2}}{\sigma^{(i-1)}} .
$$

For the average photomultiplier $\sigma \approx 4$, so that terms beyond the second can be reasonably neglected. Thus, the fluctuations in time of the output pulse of the photomultiplier are determined primarily by the transit-time dispersions in the regions between the cathode and the first dynode and between the first and second dynodes.

$$
\bar{v}_{2} \cong \frac{1}{Q}\left(\beta_{1}^{2}+\frac{\beta_{2}^{2}}{\sigma}\right) .
$$

In addition, there will be an apparent time fluctuation due to fluctuations in gain, primarily in the first stage. The apparent variance in time produced by the gain fluctuation will be denoted by $\bar{v}_{3}=\gamma^{2} / Q$, where $\gamma$ is a constant determined by the distribution of multiplication factors over a large number of processes.

To obtain the apparent variance in pulse time $(\bar{v})$, we assume, as would be true for the convolution of Gaussian distribution, that the variance resulting from the several factors involved is the sum of the individual variances, so that

$$
\begin{aligned}
\bar{v} & =\bar{v}_{1}+\bar{v}_{2}+\bar{v}_{3} \\
& =\frac{Q}{2(R \lambda)^{2}}+\frac{2 Q^{2}}{3 R(R \lambda)^{2}}+\frac{1}{Q}\left(\beta_{1}^{2}+\frac{\beta_{2}^{2}}{\sigma}+\gamma^{2}\right) \\
& =\frac{Q}{2(R \lambda)^{2}}+\frac{2 Q^{2}}{3 R(R \lambda)^{2}}+\frac{T^{2}}{Q},
\end{aligned}
$$

where $T$ is a constant characteristic of the photomultiplier.

Considering the case where $Q / R$ is small, we can easily find the value of $Q$ corresponding to minimum dispersion.

$$
\begin{aligned}
\frac{d \bar{v}}{d Q} & =\frac{1}{2(R \lambda)^{2}}-\frac{T^{2}}{Q^{2}}=0 \\
Q & =\sqrt{2} R \lambda T \\
\bar{v}_{\text {min }} & =\frac{\sqrt{2} R \lambda T}{2(R \lambda)^{2}}+\frac{T^{2}}{\sqrt{2} R \lambda T}=\frac{\sqrt{2} T}{R \lambda} .
\end{aligned}
$$

For a coincidence system using two photomultipliers, the variance of the response curve will be $2 \bar{v}$, and the width at half maximum of this curve of coincidence rate versus delay will be given by

$$
W=2.4(2 \bar{v})^{1 / 2} .
$$

When $Q / R$ is small, we can utilize the expression for $\bar{v}_{\min }$ to obtain

$$
W_{\mathrm{min}} \simeq 4\left(\frac{T}{R \lambda}\right)^{1 / 2}
$$

In practice we shall find that $Q / R=0.3$ to 0.5 , so that the value obtained from eq (3) for $W_{\min }$ will represent a lower bound-perhaps 10 to 20 percent lower than the minimum obtainable width for the resolution curve.

In order to compare the result displayed in eq (2) with experimental data obtained with a differential coincidence system, it is necessary to decide what factors determine $Q$ for a system of this type. The shorted stub technique of pulse shaping will provide a pulse for the coincidence circuit where width is approximately equal to the rise time of the original current pulse at the anode of the photomultiplier. This rise time will be determined by the dispersion in the multiplier. We are interested in the number of photoelectrons $(Q)$ that produce the secondary electron arriving at the anode during the rise of the pulse. As the rise time is determined by the dispersion in the tube, the number $(Q)$ will be proportional to the rate of production of photoelectrons, and we can write

$$
Q=K R \lambda=K^{\prime} \sqrt{2} R \lambda T .
$$

When $Q / R$ is small, the dispersion is determined by

$$
\bar{v}=K^{\prime} \frac{\sqrt{2} T}{2 R \lambda}+\frac{1}{K^{\prime}} \frac{\sqrt{2} T}{2 R \lambda}=\frac{\left(K^{\prime 2}+1\right)}{2 K^{\prime}} \frac{\sqrt{2} T}{R \lambda},
$$


and the width at half maximum of the resolution curve is

$$
4\left(\frac{K^{\prime 2}+1}{2 K^{\prime}}\right)^{1 / 2}\left(\frac{T}{R \lambda}\right)^{1 / 2}
$$

This expression exhibits a broad minimum in the neighborhood of $K^{\prime}=1$, and if $1 / 2<K^{\prime}<2$, the width of the resolution curve will be within 10 percent of the minimum obtainable with the combination of tube and phosphor.

It is of interest to consider in more detail the determination of the value of $K^{\prime}$. The current pulse at the output of the photomultiplier can be described by

$g(t)=c \int_{0}^{\infty}\left[\exp \left\{-\frac{\left(t-t_{m}\right)^{2}}{2 T^{2}}\right\}\right]\left[\exp \left\{-\lambda t_{m}\right\}\right] d t_{m}$

Where the zero of $t$ and $t_{\mathrm{m}}$ corresponds to the average time of arrival at the anode of the secondaries from a photoelectron emitted at the time of the interaction in the phosphor, $T$ measures the dispersion of the photomultiplier, and $\lambda$ is the decay constant of the phosphor.

It can be shown in a straightforward manner that

$$
\begin{aligned}
g(t)=\frac{\sqrt{2 T C} \sqrt{\pi}}{2}\{ & \left.\exp \left(\frac{\lambda T}{\sqrt{2}}\right)^{2}\right\}\{\exp (-\lambda T)\} \times \\
& \left\{1-\frac{1}{\sqrt{2 \pi}} \int_{-(\lambda T-t / T)}^{(\lambda T-t / T)} \exp \left(-\frac{\varphi^{2}}{2}\right) d \varphi\right\} .
\end{aligned}
$$

We desire to obtain an expression for the rise time $\left(t_{r}\right)$ of this current pulse. At this time $d g / d t=0$. This condition yields the equation

$$
\begin{aligned}
{\left[1-\frac{1}{\sqrt{2 \pi}} \int_{-\left(\lambda T-t_{r} / T\right)}^{\left(\lambda T-t_{r} / T\right)} \exp \left(-\frac{\varphi^{2}}{2}\right) d \varphi\right] } \\
=\frac{2}{\lambda T_{\sqrt{\pi 2}}} \exp \left\{-\frac{\left(\lambda T-t_{r} / T\right)^{2}}{2}\right\} .
\end{aligned}
$$

If $\lambda$ and $T$ are known, this equation can easily be solved for $t_{r}$ by numerical procedures with the aid of Tables of Normal Probability Functions [5], which tabulates both functions. Best agreement with data presented in the next section is obtained by assuming a value of $T=1.8 \times 10^{-9}$ sec in normal operation of the photomultiplier at $1,600 \mathrm{v}$.

\subsection{Comparison with Experiment}

Calculations have been made of the expected values of $\left(t_{r}\right)$ for stilbene and terphenyl-toluene solution, assuming the above value $\left(1.8 \times 10^{-9} \mathrm{sec}\right)$ for $T$ and $1 / \lambda=6 \times 10^{-9}$ sec and $1 / \lambda=2.5 \times 10^{-9}$ sec. respectively. For the two cases,

$$
\begin{aligned}
& t_{r}=1.27 T \quad \text { (for stilbene) } \\
& t_{r}=0.86 T \quad \text { (for terphenyl-toluene). }
\end{aligned}
$$

The maximum current occurs a time $t_{r}$ after the average arrival time of the secondaries produced by a photoelectron ejected at the time of interaction in the phosphor. The photoelectron charge, $Q$, contributing to the rise of the pulse is, on the average

$$
D=R\left\{1-\exp \left(-\lambda t_{r}\right)\right\},
$$

which yields

$$
\begin{array}{ll}
Q=0.76 \sqrt{2} R \lambda T \quad \text { (for stilbene) } \\
Q=0.45 \sqrt{2} R \lambda T \quad \text { (for terphenyl-toluene). }
\end{array}
$$

In the case of stilbene, we neglect the term in $Q^{2}$ in eq (1) to obtain from eq (3) a lower limit on the width at half maximum of the coincidence response curve. We assume, in accordance with the work of Sangster [7], that stilbene gives 50 percent of the photoelectron yield of anthracene in a 5819 photomultiplier. The yield of anthracene is half that of $\mathrm{NaI}$ measured by Hofstadter [8] to be about one photoelectron per kilo electron volt. For cobalt-60 gamma rays, the average energy of the Compton electrons producing pulses accepted by the pulse height selectors is $900 \mathrm{kev}$. For stilbene we expect, therefore, 225 photoelectrons on the average. Using this value for $R$, we obtain a lower limit of $8.8 \times 10^{-10}$ sec for the width at half maximum of the resolution curve. The expected value given by formulas (1) and $(2)$ is $9.7 \times 10^{-10} \mathrm{sec}$. The results of a measurement with the differential coincidence system are shown in figure 9, and confirm this value to within

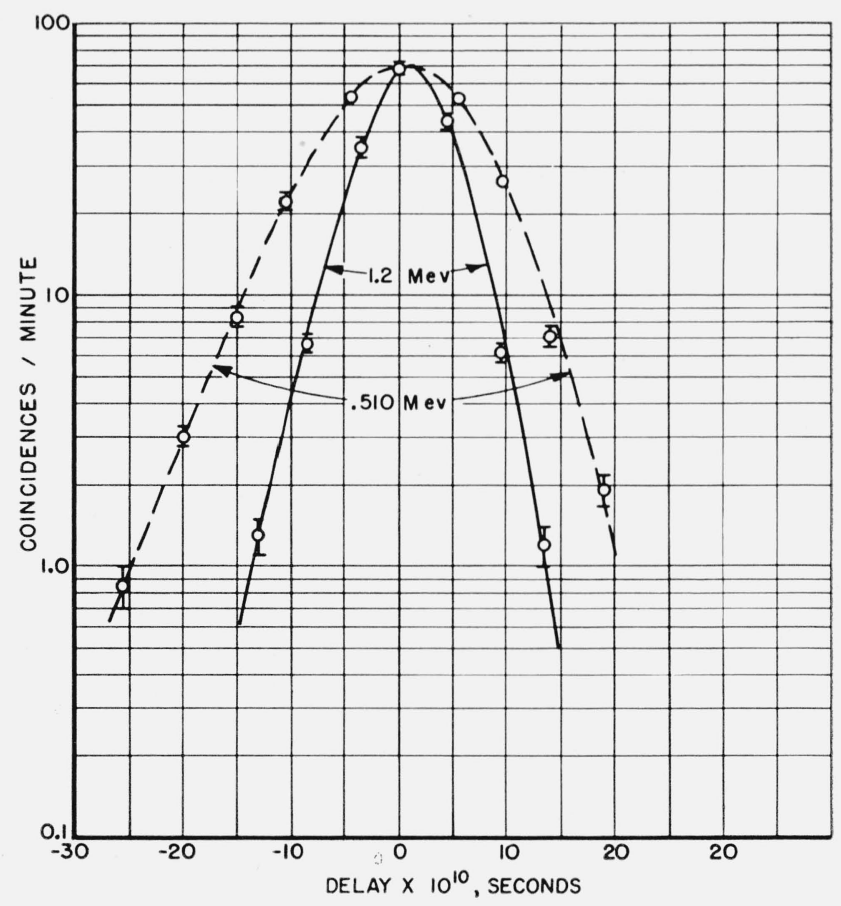

Figure 9. Effect on the response of the differential coincidence system of figure 5 produced by a variation in the number of photons emitted by the phosphor.

5819 photomultipliers and stilbene crystals were used. 
10 percent, which is as good as can be expected in view of the uncertainties in the parameters involved. Figure 9 also shows the results of a measurement with annihilation radiation. In accordance with formula (2), we would expect a ratio of widths at half maximum of

$$
\frac{W_{\text {Annihilation }}}{W_{\mathrm{Co}^{60}}}=\left(\frac{R_{\mathrm{Co}^{60}}}{R_{\text {Annihilation }}}\right)^{1 / 2}=1.75
$$

because the average energy corresponding to the accepted pulses from the annihilation spectrum is $300 \mathrm{kev}$. Using this ratio and the expected width of the resolution curve for cobalt-60 radiation, we find that the expected width of the low-energy curve is $17 \times 10^{-10}$ sec. The observed width is $16 \times 10^{-10}$ sec.

In the case of the terphenyl-toluene scintillator, it is desirable to include all terms of eq (1) in estimating a lower limit for $W$, as $Q / R$ is close to 0.5 . The value of $R$ is chosen in accordance with the work of Kallman and Furst [9] as $0.57 R_{\text {stilbene. }}$ For $\mathrm{Co}^{60}$ gammas, $R=130$ is used. The expression (1) for $\bar{v}$ can be minimized for our particular value of $\lambda T$ and, with (2), yields $W_{\min }=8.9 \times 10^{-10}$ sec. The expected value of $W$ is given by letting $Q=0.45 \sqrt{2} R \lambda T$ in the expression for $\frac{v}{v}$. This gives $W_{\text {calc }}=9.1 \times 10^{-10}$ sec. The experimental curve is shown as a dotted curve in figure 11 , and yields $W_{\text {obs }}=10 \times 10^{-10} \mathrm{sec}$. For annihilation radiation, $R=130 / 3$. The value of $W$ calculated from eq (1) and (2) is $16 \times 10^{-10}$ sec. The experimental curve is shown in figure 10 along with that for stilbene, and gives $W_{\mathrm{obs}}=15 \times 10^{-10}$ sec.

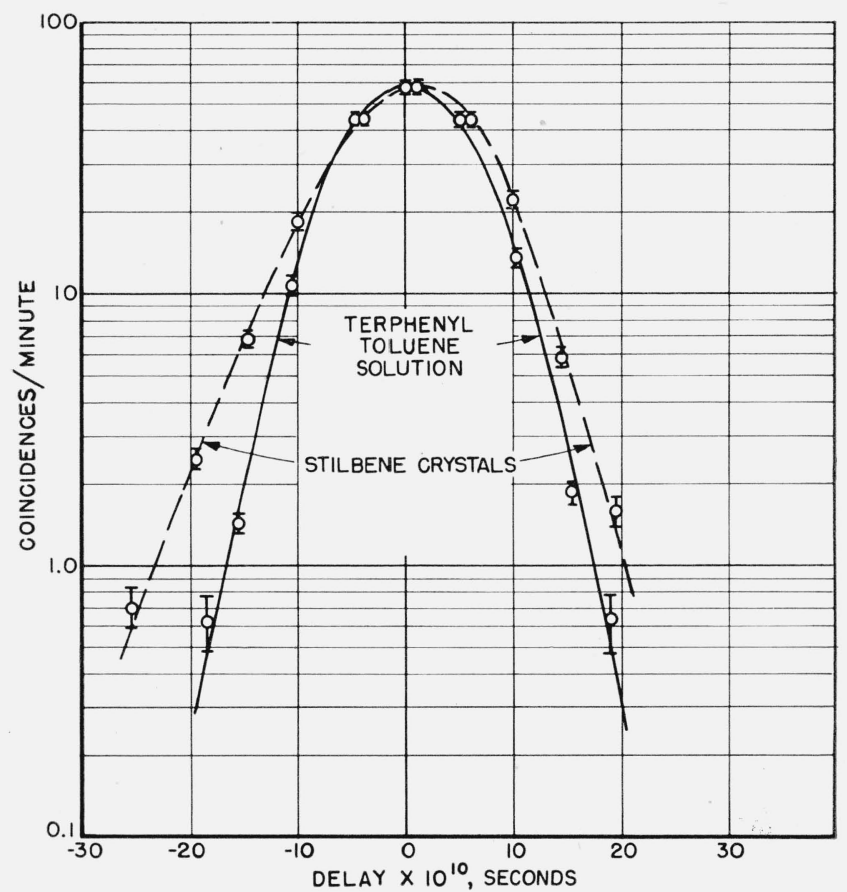

FIGURE 10. Effect on the response of the differential coincidence system of figure 5 produced by a difference in the time distribution of photons incident on the photomultiplier using annihilation radiation.
In order to observe the effect of changing $T$, the voltage across the eathode to first dynode gap was increased by a factor of 4 and that from dynode 1 to dynode 2 by a factor of 2 . From the geometries and voltages involved, it appears that $\beta_{1} \approx \beta_{2}$. Neglecting $\gamma$, we have

$$
\frac{T_{2}^{2}}{T_{1}^{2}}=\frac{\beta^{2}+\frac{\beta^{2}}{4}}{4 \beta^{2}+\frac{\beta^{2}}{2}}=\frac{1}{3.6},
$$

because the quantity $\beta$ is a measure of the meansquare spread in transic times and should be inversely proportional to the square root of the voltage. Thus the value of $T_{2}$ is $\left(1.8 \times 10^{-9}\right) / \sqrt{3.6}=9.5 \times 10^{-10}$ sec.

Using this value for $T$, we can calculate with the aid of eq (4) the rise time of the pulse on the anode of the tube. We find

$$
\begin{aligned}
& t_{r}=1.16 T_{2}, \\
& Q=0.95 R \lambda T_{2} .
\end{aligned}
$$

$T_{2}$ is sufficiently short to neglect the second-order term in calculating the lower limit on the width of the resolution curve. For cobalt-60 gamma rays on terphenyl-toluene we obtain $W_{\mathrm{min}}=5.4 \times 10^{-10} \mathrm{sec}$. The calculated value of $W$, using eq (1) and (2), is $6.1 \times 10^{-10}$ sec.

The observed value of $W$ obtained from the experimental curve of figure 11 is $6.2 \times 10^{-10}$ sec.

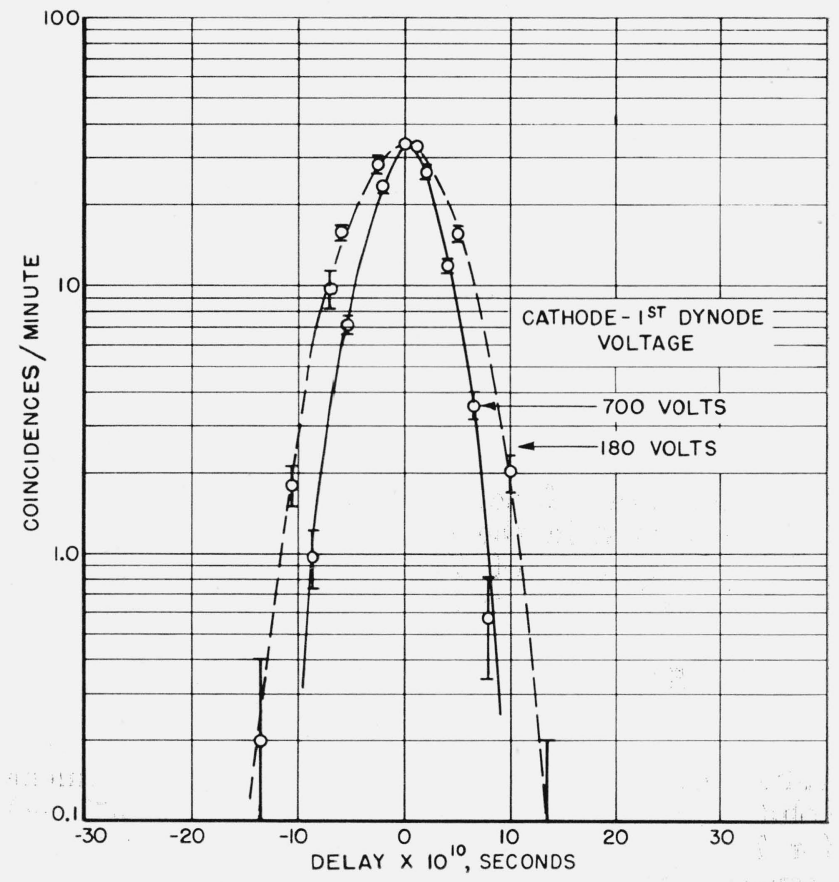

FIGURE 11. Effect on the response of the differential coincidence system of figure 5 produced by a change in the transit time in the photocathode-to-dynode region of the photomultiplier.

5819 photomultipliers and terphenyl-toluene scintillators were used with $\mathrm{Co}^{60} \gamma$ radiation. 
TABLE 1. Width at half-maximum of curves of coincidence rate versus delay

\begin{tabular}{|c|c|c|c|c|c|}
\hline Scintillator & Energy & Voltage & $W_{\min }$ & $W_{\text {ealo }}$ & $W_{\text {obs }}$ \\
\hline $\begin{array}{l}\text { Stilbene } \\
\text { Do } \\
\text { Terphenyl-toluene } \\
\text { Do } \\
\text { Do }\end{array}$ & $\begin{array}{l}k e v \\
900 \\
290 \\
900 \\
290 \\
900\end{array}$ & $\begin{array}{c}v \\
1,600 \\
1,600 \\
1,600 \\
1,600 \\
2,400\end{array}$ & $\begin{array}{l}s e c \\
8.8 \times 10^{-10} \\
15.5 \\
8.9 \\
15.5 \\
5.4\end{array}$ & $\begin{aligned} & s e c \\
& 9 . 7 \times 10^{-10} \\
& 17.0 \\
& 9 . 1 \\
& 16.0 \\
& 6.1\end{aligned}$ & $\begin{array}{l}\quad s e c \\
9.0 \times 10^{-10} \\
16.0 \\
10.0 \\
15.0 \\
6.2\end{array}$ \\
\hline
\end{tabular}

The results of these measurements are summarized in table 1 . The agreement between calculated and observed widths of the resolution curves is excellent in view of the uncertainty in the values for $R$ and $\lambda$. These values were obtained from the literature. The values for $R$ are considerably more in doubt, but the relative values should be within 10 to 15 percent. The value of the single parameter, $T$, was adjusted to give best agreement between the calculated and experimental values of $W$. An error in the absolute value of $R$ would be reflected in this quantity. The value of $1.8 \times 10^{-9} \mathrm{sec}$ obtained from this quantity does not appear to be unreasonable. Moreover, the consistency of the results suggests that the calculation accurately represents the behavior of the coincidence system, and the conclusion may be drawn that in all cases observed the experimental resolution was very close to the minimum obtainable with the combination of phosphor and phototube used.

\section{Multiplex Coincidence System}

The attainment of systems having better resolution awaits the development of new phototubes capable of operating at very high voltages in the early stages and the discovery of faster phosphors, or requires the use of a different combination of crystal and multiplier. The latter alternative is illustrated by the following suggestion. If there are fluctuations in time of the pulses from the detectors for truly coincident events, the influence of these fluctuations on data indicating delayed radiation can be reduced by using two photomultipliers viewing each phosphor and requiring a delayed coincidence to be registered simultaneously in two independent coincidence circuits. Delayed radiation will produce a delayed coincidence in both channels, whereas a randomly occurring fluctuation is much less likely to have the same value in both sets of detectors at the same time. In fact, it has been experimentally verified that the curve of counts versus time for coincident radiation events falls off as the product of the curves for the individual coincidence circuits. The drawback on this procedure is that if two photomultipliers are used, a reflector must be removed, and the amount of light collected by each photomultiplier is less than if only one tube is used. This decrease in light intensity corresponds to a decrease in $R$ and has the same effect as a decrease in energy of the original radiation. That is, the fluctuations in the response of the individual coincidence systems are increased.

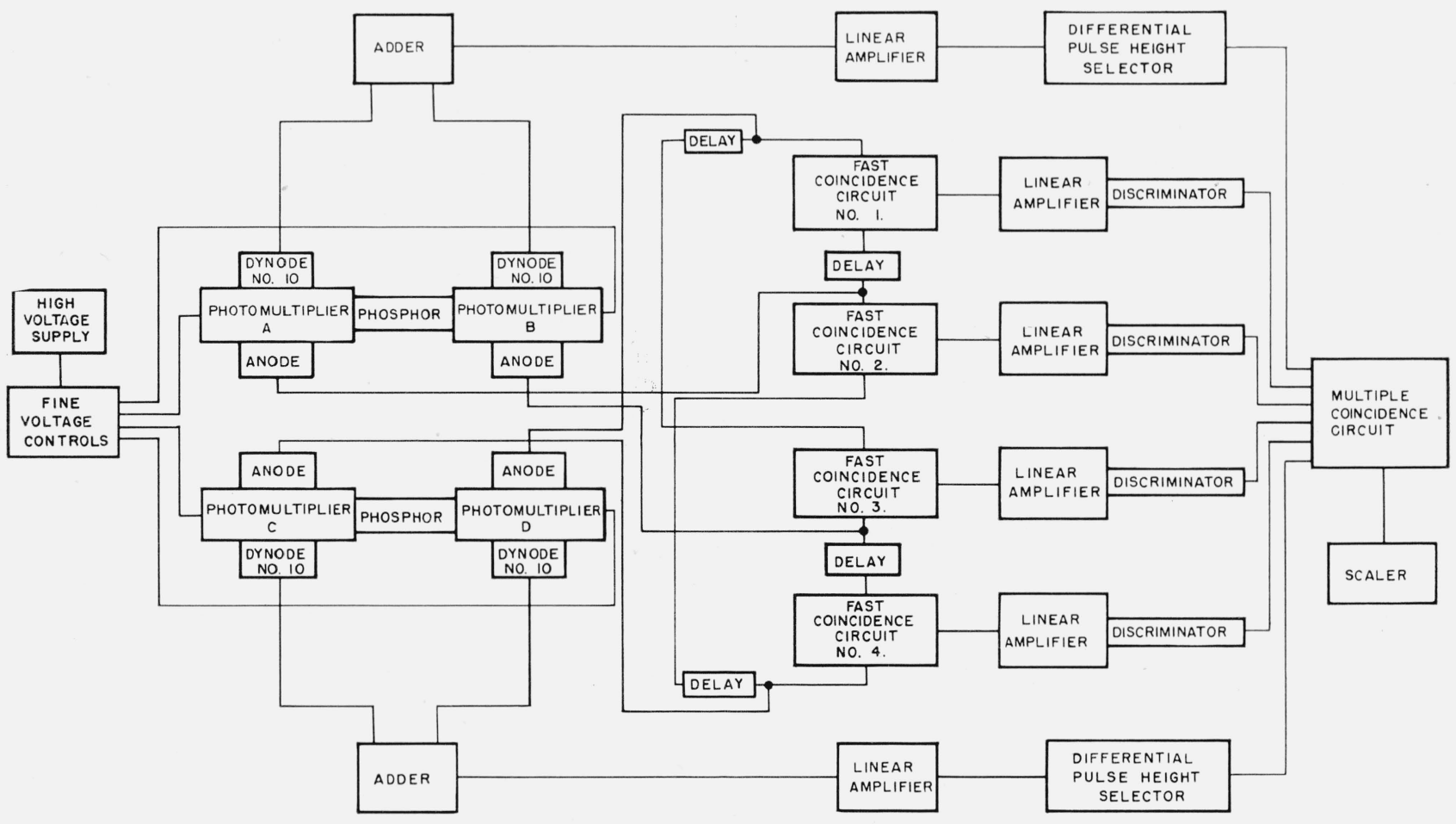

FIGURE 12. Block diagram of multiplex coincidence system devised to minimize the effects of the time fluctuations in the scintillation counters. 
A system based on this principle has been designed and constructed. It consists of two phosphors, each viewed by two photomultipliers. In this system, RCA 6199 photomultipliers were used because of their high gain and low noise. Four of the six tubes available were operated successfully at $2,000 \mathrm{v}$ and produced larger pulses than the selected 5819 photomultipliers used previously. The pulses from each phototube are fed to the inputs of two fast double-coincidence circuits, so that four of these circuits are used in the system. To minimize the effect of pulse width, the cabling is arranged so that, for zero delay and coincident radiation, each pulse arrives earlier than its counterpart at one coincidence circuit and later at the other. A time fluctuation in either direction will then eliminate the coincidence in 1 of the 2 circuits. The outputs of the fast coincidence circuits drive four inputs of a sixfold coincidence circuit. The other two inputs are driven by the outputs of two pulse-height selectors, each of which analyzes the summed pulses from the last dynodes of the photomultipliers viewing one of the phosphors.

A block diagram of this system is shown in figure 12. The system has been tested by means of the coincident gamma rays from $\mathrm{Co}^{60}$ and from positron annihilation. The resolution curves are shown in figure 13. The resolution obtained with this system is about the same as that obtained with one differen-

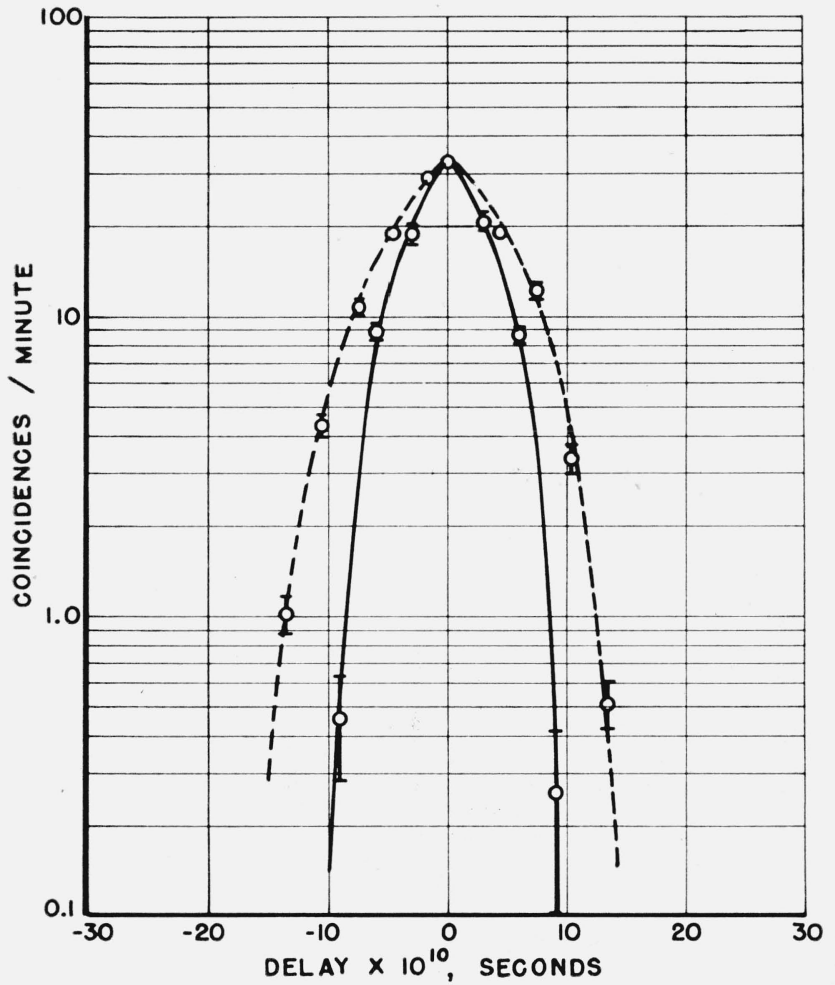

FIgURE 13. Response of the multiplex coincidence system of figure 12 to pulses produced by coincident gamma rays incident on terphenyl in toluene liquid scintillators.

$\mathrm{Co}^{60}$ gamma rays; ........, annihilation radiation. 6199 photomul-

tipliers were used. tial coincidence system and 5819 photomultipliers operated at $2,400 \mathrm{v}$. From the standpoint of stability, however, the new system has been much more satisfactory than the old one, primarily because of a noticeable reduction in fatigue of the photomultipliers.

\section{Applications of Coincidence Techniques}

The mode of operation of high-speed coincidence circuitry is determined by the specific requirements of the problem at hand. If high efficiency is required in the detection of a coincidence, a sacrifice in resolution must be made because of the inherent time fluctuations in the detectors. Such a situation is encountered, for example, in the absolute calibration of radiation sources by coincidence methods. In this case, the system must operate with a constant known efficiency. An efficiency of 100 percent has been found to be the easiest to measure and hold constant, and a coincidence system has been de. veloped for the absolute calibration of cobalt-60 sources by the method of gamma-gamma coincidences operating at this efficiency. For this application, the differential coincidence technique was not feasible because, with terphenyl-toluene scintillators, the maximum fluctuations in pulse time are of the order of magnitude of the pulse width. Because of the fluctuations in pulse time, it was necessary to accept a resolution $T=1.5+10^{-9}$ sec in order to achieve 100 percent efficiency. Under these conditions we are able to determine the disintegration rate of millicurie sources of cobalt-60 to an accuracy of 1 percent.

If the problem is primarily one of measuring short time intervals between radiations, on the other hand, and less than 100 percent efficiency is tolerable, it is preferable to operate the coincidence system in such a way as to provide maximum resolution. In this application, it is possible to measure time intervals considerably shorter than the width of the resolution curve for the system. Bay [10] and Newton [11] have discussed some methods for analysis of the data obtained in such measurements. It can be shown that if the time delays are the result of the exponential decay of an intermediate state excited by the first radiation, the curve of counts versus artificial delay is given by

$$
\begin{aligned}
C(d)=A S \sqrt{\pi}\left(\frac{\alpha}{2 K}\right)\left[\exp \left\{\left(\frac{\alpha}{2 K}\right)^{2}\right\}\right][\exp \{-\alpha d\}] \times \\
{\left[1-\frac{1}{\sqrt{2 \pi}} \int_{-\sqrt{2}\left(\frac{\alpha}{2 K}-K d\right)}^{\sqrt{2}\left(\frac{\alpha}{2 K}-K d\right)} \exp \left\{-\frac{\phi^{2}}{2}\right\} d \phi\right], }
\end{aligned}
$$

where $A$ is a constant that depends on the geometry and resolution of the systam, $S$ is the source strength, $\alpha$ is the decay constant of the intermediate state, and the prompt coincidence resolution curve of the system for radiations of the same energy is described by $C(d)=C_{o} e^{K^{2} d^{2}}$. The function contained in brackets has been tabulated in [5] so that it is 
possible to calculate curves to be compared with the experimental curve and to determine the value of $\alpha$ that gives a best fit. From a prompt coincidence resolution curve such as the solid curve of figure 13 , it is possible to measure lifetimes of the order of $10^{-10}$ sec to an accuracy of the order of $10^{-11}$ sec. These techniques have been applied to data obtained from the annihilation of positrons in metals and differences have been determined of $4 \times 10^{-11}$ sec between mean lives to a precision of $1 \times 10^{-11}$ sec. Details of this work will be published elsewhere.

\section{Conclusions}

The data and analysis presented here indicate that the circuitry and techniques described enable one to obtain coincidence resolutions very close to the minimum to be expected for the combinations of scintillator and photomultiplier presently available. The analysis of the detectors and circuitry takes account of the fluctuations in emission time of the photons from the phosphor and assumes Gaussian distributions for the interstage transit times in the photomultipliers. The time of occurrence of a pulse is considered to be the average of the arrival times at the anode of the electrons that contribute to the rise of the output pulse. This analysis results in the satisfactory prediction of the magnitude of the resolution and the effect on the resolution produced by a variation of tube voltage, phosphor decay time, or energy of exciting radiation. The data indicate that the root-mean-square deviation of the transit time in the cathode-to-dynode region of the 5819 photomultiplier is $1.8 \times 10^{-9}$ sec when the voltage across the gap is $180 \mathrm{v}$.
Fluctuations in photon-emission time and electron-transit time limit the time resolution obtainable with a scintillation counting system, so that the use of circuitry having too short a resolution results in reduced efficiency for the detection of coincident radiation. For $1-\mathrm{Mev}$ gamma rays the limiting resolution for 100-percent efficiency is approximately $1.5 \times 10^{-9}$ sec. Operation of the system at reduced efficiency enables one to measure time intervals of the order of $10^{-10}$ sec between radiations to an accuracy of 10 percent.

\section{References}

[1] S. DeBenedetti and H. J. Richings, Rev. Sci. Instr. 23, $27(1952)$

[2] Z. Bay, Phys. Rev. 83, 242 (1951).

[3] R. F. Post and L. I. Schiff, Phys. Rev. 80, 1113 (1950).

[4] G. A. Morton, Nucleonics 10, 39 (1952).

[5] Tables of normal probability functions, NBS Applied Mathematics Series 23 (1953).

[6] R. F. Post, Phys. Rev. 78, 80 (1950); 79, 735 (1950).

[7] R. C. Sangster, Tech. Report No. 55 (Research Laboratory for Electronics, Mass. Inst. Technology, January 1952).

[8] R. Hofstadter and J. A. MeIntyre, Phys, Rev. 80, 631 (1950). See also, J. I. Hopkins, Rev. Sci. Instr. 22, 29 (1951).

[9] M. Furst and H. Kallmann, Phys. Rev. 85, 816 (1952).

[10] Z. Bay, Phys. Rev. 77, 419 (1950).

[11] T. D. Newton, Phys. Rev. 88, 490 (1950).

Washington, April 13, 1956. 